\title{
Transformations among CE-CVM model parameters for multicomponent systems
}

\author{
B NAGESWARA SARMA and SHRIKANT LELE* \\ Department of Metallurgical Engineering, Institute of Technology, Banaras Hindu University, Varanasi 221 005, India
}

MS received 24 June 2004; revised 24 February 2005

\begin{abstract}
In the development of thermodynamic databases for multicomponent systems using the cluster expansion-cluster variation methods, we need to have a consistent procedure for expressing the model parameters (CECs) of a higher order system in terms of those of the lower order subsystems and to an independent set of parameters which exclusively represent interactions of the higher order systems. Such a procedure is presented in detail in this communication. Furthermore, the details of transformations required to express the model parameters in one basis from those defined in another basis for the same system are also presented.
\end{abstract}

Keywords. Cluster expansion and cluster variation methods; phase equilibria; thermodynamics of materials.

\section{Introduction}

The cluster variation method (CVM) proposed by Kikuchi (1951) provides a systematic hierarchy of approximations for obtaining configurational entropy of alloy systems by considering local order as accurately as desired in terms of increasingly larger atomic clusters. The potential of CVM came to be well recognized ever since van Baal (1973) demonstrated the effect of including tetrahedral multi-atom interactions (in terms of a set of adjustable parameters) in the configurational energy expression on the topology of the phase diagram of a prototypical binary $f c c$ ordering system. The CVM is also shown to subsume many of the earlier models in its class (such as BraggWilliams-Gorsky and Bethe approximations, quasi-chemical model, etc) as lower levels of the general hierarchy of the method. Sanchez and de Fontaine (1978) introduced multi-site correlation functions that form a natural set of independent variables defining the dimensionality of the system, in terms of which, the thermodynamic potentials can be expressed. Sanchez et al (1984) subsequently proved that any function depending on atomic configuration (such as configurational energy) could be rigorously expanded as a bilinear sum of the products of cluster functions (expectation values are correlation functions) and their respective cluster expansion coefficients (CECs), since the former form a set of complete and orthonormal basis functions at the chosen level of cluster approximation. This powerful property is utilized in the method of cluster expansions (CE) to obtain the configurational energy expression, which, when combined with CVM makes it very versatile and eminently suitable for optimization

*Author for correspondence (slele@ satyam.net.in) purposes as well as in the use of first principles electron energy calculations for the determination of phase equilibria (Asta et al 1993). The developments in and the applications of CVM have been extensively reviewed from time to time (de Fontaine 1979, 1994; Inden and Pitsch 1991; Saunders and Miodownik 1998).

In the formulation of CE-CVM for multicomponent systems, the correlation functions and the CECs corresponding to the end member subsystems will have to be obtained from those defined in the basis chosen for the multicomponent system and vice versa in a consistent manner, details of which are presented in this communication. The analysis is illustrated with reference to disordered ternary $b c c$ structures in tetrahedron approximation of CE-CVM. Specifically, the procedure for obtaining ternary CECs from those of the end member subsystems will be illustrated. This is very useful for developing databases of multicomponent systems, which are consistent with their subsystems. As part of this we shall also provide the details of the basis transformations from, say, orthogonal to a nonorthogonal basis etc within a given (sub)system.

\section{Cluster algebra}

As usual in CVM, at first a motif (a set of nearby sites in the structure, in this case, 4 sites forming a tetrahedron) is chosen and atomic clusters/subclusters are obtained by populating these sites with the atomic species in all possible ways. The probabilities of occurrence of these clusters/subclusters are the cluster variables. However, all of these variables are not independent. Such an independent set of variables can be obtained in terms of multi-site correlation functions. A detailed procedure for obtaining these is given in a didactic manner by Inden and Pitsch (1991). For the case of a $k$-component system, the following basic 
equation relates site operators $s 1 i, s 2 i, \ldots, s k i$ and site occupation operators $p_{i}^{A}, p_{i}^{B}, \ldots, p_{i}^{K}$ defined for the site $i$.

$\left[\begin{array}{c}1 \\ s 1 i \\ s 2 i \\ \cdot \\ \cdot \\ \cdot \\ s k i\end{array}\right]=\left[\begin{array}{ccccc}1 & 1 & 1 & \ldots & 1 \\ -1 & 1 & 0 & \ldots & 0 \\ -1 / 2 & -1 / 2 & 1 & \ldots & 0 \\ \cdot & \cdot & . & \ldots & \cdot \\ \cdot & \cdot & . & \ldots & \cdot \\ \cdot & \cdot & \cdot & \ldots & \cdot \\ -1 /(k-1) & -1 /(k-1) & -1 /(k-1) & \ldots & 1\end{array}\right]\left[\begin{array}{c}p_{i}^{A} \\ p_{i}^{B} \\ p_{i}^{C} \\ \cdot \\ \cdot \\ \cdot \\ p_{i}^{K}\end{array}\right]$.

The square matrix appearing in (1) is called the basis matrix $A$ for a $K$ component system. This is obtained from that for a $(K-1)$ component system by augmenting with the appropriate $K$ th row and $K$ th column. Note that the row-vectors of the above basis matrix $A$ are orthogonal, since the product of the matrix with its transpose is a diagonal matrix. The matrix can, however, be made orthogonal by suitable scaling of the row vectors. This is not necessary for our purposes here. We note that the multicomponent basis utilized by Inden and Pitsch is not orthogonal. It is, therefore, suggested that this basis be used as a standard for database purposes. Sanchez et al (1984) and Wolverton and de Fontaine (1994) suggested an orthogonal basis in terms of Chebychev polynomials. It may be noted that except for binary and ternary systems, the two bases are not the same.

For the case of a ternary system, we have

$$
\left[\begin{array}{c}
1 \\
s 1 i \\
s 2 i
\end{array}\right]=\left[\begin{array}{ccc}
1 & 1 & 1 \\
-1 & 1 & 0 \\
-1 / 2 & -1 / 2 & 1
\end{array}\right]\left[\begin{array}{l}
p_{i}^{A} \\
p_{i}^{B} \\
p_{i}^{C}
\end{array}\right] .
$$

Representing the square matrix in (2) by $A$ and its inverse by $R$, we can write the linear relations among cluster variables and correlation functions (as given by Inden and Pitsch, in their equations (9-10)). In the formulation of CE-CVM for disordered ternary $b c c$ structures under tetrahedron approximation, we consider a tetrahedron motif whose vertices are labeled by 1, 2, 3 and 4. There are four first neighbour pairs (viz. 12, 14, 23 and 34) and two second neighbour pairs (viz. 13 and 24) present in the motif. All the triangle submotifs (viz. 123, 124, 134 and 234) are crystallographically identical. The averages of the site operators $(\langle s 1 i\rangle$ and $\langle s 2 i\rangle)$ and their products corresponding to the motif/submotif sites are called the correlation functions. There are a total of 18 correlation functions consisting of three first neighbour pairs (typified by $u t[1]=$ $\langle s 11 * s 12\rangle, u t[2]=\langle s 12 * s 21\rangle$ and $u t[3]=\langle s 21 * s 22\rangle)$, three second neighbour pairs (typified by $u t[4]=\langle s 11 * s 13\rangle$, $u t[5]=\langle s 13 * s 21\rangle$ and $u t[6]=\langle s 21 * s 23\rangle$ ), six triangles (typified by $u t[7]=\langle s 11 * s 12 * s 13\rangle, u t[8]=\langle s 12 * s 13 * s 21\rangle$, $u t[9]=\langle s 11 * s 13 * s 22\rangle, u t[10]=\langle s 13 * s 21 * s 22\rangle, u t[11]=$ $\langle s 12 * s 21 * s 23\rangle$ and $u t[12]=\langle s 21 * s 22 * s 23\rangle)$ and six tetrahedra (typified by $u t[13]=\langle s 11 * s 12 * s 13 * s 14\rangle$, $u t[14]=\langle s 12 * s 13 * s 14 * s 21\rangle, u t[15]=\langle s 13 * s 14 * s 21 * s 22\rangle$, $u t[16]=\langle s 12 * s 14 * s 21 * s 23\rangle, u t[17]=\langle s 14 * s 21 * s 22 * s 23\rangle$ and $u t[18]=\langle s 21 * s 22 * s 23 * s 24>$ ). The two point correlation functions are related to composition variables in the following manner. $u t[19]=\langle s 1 i\rangle=-\left\langle p_{i}^{A}\right\rangle+\left\langle p_{i}^{B}\right\rangle=$ $-x_{A}+x_{B}$ and $u t[20]=\langle s 2 i\rangle=-x_{A} / 2-x_{B} / 2+x_{C}$ [see (2)]. Further, there are a total of 54 crystallographically distinct cluster configurations (variables) including three composition variables. All these cluster variables can be expressed as linear combinations of the correlation functions and the exact nature of this linear transformation is dependent on $A$ and its inverse $R$.

\section{Relations among ternary CECs and binary CECs}

As indicated earlier, we need to express the CECs for a ternary system in terms of those for its binary subsystems. This enables us to use the CECs of binary subsystems which might be obtained separately by, say, optimization of thermodynamic and phase equilibria data for the binary subsystems. The procedures for accomplishing the same are outlined below. We note in passing that Kikuchi (1977) and Kikuchi et al (1977), who first formulated CVM for a ternary system, use energy coefficients for all the clusters corresponding to the maximal motif (tetrahedron approximation for ternary $f c c$ phase). Such a formulation determines all the cluster variables (54 in the present case) in the minimization step, by using 'natural iteration method', and is not very efficient compared to the determination of correlation functions (18 in the present case) using 'Newton-Raphson method'. We are, therefore, presenting the details necessary for the latter case in a systematic manner. These can thus be extended to the general case of multicomponent systems.

At first, we obtain the enthalpy of mixing for the ternary phase as follows. As pointed out earlier, Sanchez et al (1984) have shown that any function of configuration, for example, internal energy $U$ of an alloy, can be expressed as a bilinear sum of the products of the CECs et $[i]$ and the corresponding correlation functions $u t[i]$. Thus

$$
U=\sum_{i=1}^{21} e t[i] m[i] u t[i] .
$$

Here, $m[i]$ are the multiplicities of a motif/submotif corresponding to the respective correlation functions. The multiplicities are defined as the number of symmetry-equivalent motifs/submotifs per site present in the structure. The multiplicities for first neighbour pairs, second neighbour pairs, triangles, tetrahedra and points are respectively, $4,3,12,6$ and 1 . For the invariant empty cluster, the multiplicity and its correlation function are unity (independent of the basis). The value of the CEC corresponding to the 
empty cluster thus serves as the reference level for the expansion. From (2), we have for pure $A,\langle s 1 i\rangle=-1$, $\langle s 2 i\rangle=-1 / 2$; for pure $B,\langle s 1 i\rangle=1,\langle s 2 i\rangle=-1 / 2$ and for pure $C,\langle s 1 i\rangle=0,\langle s 2 i\rangle=1$. Substituting these successively in (3) yields the internal energies UA, UB and $\mathrm{UC}$, respectively for pure $A, B$ and $C$. Thus, we can obtain the internal energy of mixing $\Delta U 3$ as follows.

$$
\begin{aligned}
& \Delta U 3=U-x_{A} * U A-x_{B} * U B-x_{C} * U C= \\
& (-8 * \operatorname{et}[1]) / 3+4 * u t[1] * \operatorname{et}[1]+\left(8^{*}<s 21>* e t[1]\right) / \\
& 3+2 *<s 11>* \operatorname{et}[2]+4 * u t[2] * \operatorname{et}[2]-2 * \operatorname{et}[3]- \\
& 2 *<s 21>* e t[3]+4 * u t[3] * \operatorname{et}[3]-2 * \operatorname{et}[4]+ \\
& 3 * u t[4] * e t[4]+2 *<s 21>\operatorname{et}[4]+(3 *<s 11> \\
& * e t[5]) / 2+3 * u t[5] * e t[5]-(3 * e t[6]) / 2- \\
& (3 *<s 21>* e t[6]) / 2+3 * u t[6] * \operatorname{et}[6]- \\
& 12 *<s 11>* \operatorname{et}[7]+12 * u t[7] * \operatorname{et}[7]+4 * \operatorname{et}[8]- \\
& 4 *<s 21>* e t[8]+12 * u t[8] * e t[8]+4 * e t[9]- \\
& 4 *<s 21>* \operatorname{et}[9]+12 * u t[9] * \operatorname{et}[9]- \\
& 3 *<s 11>* \operatorname{et}[10]+12 * u t[10] * \operatorname{et}[10]- \\
& 3 *<s 11>* e t[11]+12 * u t[11] * \operatorname{et}[11]-3 * \operatorname{et}[12]- \\
& 9 *<s 21>* \operatorname{et}[12]+12 * u t[12] * \operatorname{et}[12]- \\
& 4^{*} \operatorname{et}[13]+6^{*} u t[13]^{*} \operatorname{et}[13]+4^{*}<s 21>* \operatorname{et}[13]+ \\
& 3 *<s 11>* e t[14]+6 * u t[14] * e t[14]- \\
& \text { et }[15]+\langle s 21\rangle^{*} \operatorname{et}[15]+6^{*} u t[15]^{*} \operatorname{et}[15]- \\
& \text { et }[16]+\langle s 21\rangle * \operatorname{et}[16]+6^{*} u t[16] * \operatorname{et}[16]+ \\
& (3 *<s 11>* \operatorname{et}[17]) / 4+6 * u t[17] * \operatorname{et}[17]- \\
& (9 * e t[18]) / 4-(15 *<s 21>* \operatorname{et}[18]) / 4+ \\
& \text { 6*ut }[18]^{*} e t[18] \text {. }
\end{aligned}
$$

The internal energy of mixing for the $A-B$ subsystem can be obtained by making appropriate substitutions in this expression. The same can also be obtained directly from the formulation for the binary system. A term-wise comparison of these two expressions yields relations among the ternary CECs and the binary CECs for $A-B$ subsystem. The details are given below.

For the binary subsystem $A-B, p_{i}^{C}=0$. Thus, $s 2 i=-1 / 2$. By making these substitutions in the definitions of correlation functions, we have

$$
\begin{aligned}
& <s 12 * s 21>=-1 / 2<s 12>=-1 / 2\left(x_{A}+x_{B}\right)=-1 / 2, \\
& <s 21 * s 22>=1 / 4, \\
& <s 13 * s 21>=-1 / 2<s 13>=-1 / 2\left(x_{A}+x_{B}\right)=-1 / 2,
\end{aligned}
$$

etc. Making these substitutions in (4), we obtain the following expression for the internal energy of mixing for the system $A-B$.

$$
\begin{aligned}
\Delta U 3 A B= & (-4+4 * u t[1]) * \operatorname{et}[1]+(-3+3 * u t[4]) \\
& * \operatorname{et}[4]+(-12 *<s 11>+12 * u t[7]) * \operatorname{et}[7]+ \\
& (6-6 * u t[1]) * \operatorname{et}[8]+(6-6 * u t[4]) * \operatorname{et}[9]+ \\
& (-6+6 * u t[13]) * \operatorname{et}[13]+(3 *<s 11>- \\
& 3 * u t[7]) * \operatorname{et}[14]+(-3 / 2+(3 * u t[1]) / 2) * \\
& \operatorname{et~}[15]+(-3 / 2+(3 * u t[4]) / 2) * \operatorname{et}[16] .
\end{aligned}
$$

In order to obtain the internal energy of mixing for the $A-B$ system directly from the formulation for the binary system, the appropriate binary basis can be found by sett- ing $p_{i}^{C}=0$ in (2). This results in an orthogonal basis given by

$$
\left[\begin{array}{c}
1 \\
s 1 i
\end{array}\right]=\left[\begin{array}{cc}
1 & 1 \\
-1 & 1
\end{array}\right]\left[\begin{array}{c}
p_{i}^{A} \\
p_{i}^{B}
\end{array}\right] .
$$

There are four correlation functions (viz. $u A B[1]=$ $\langle s 11 * s 12\rangle, u A B[2]=\langle s 11 * s 13\rangle, u A B[3]=\langle s 11 * s 12 * s 13\rangle$ and $u A B[4]=\langle s 11 * s 12 * s 13 * s 14\rangle$ ), apart from the point correlation function $\langle s 11\rangle=-x_{A}+x_{B}$. The internal energy of mixing for the binary case is given by

$$
\begin{aligned}
\Delta U 2 A B= & (-4+4 * u A B[1]) * e A B[1]+(-3+3 * u A B[2]) \\
& * e A B[2]+(-12 *<s 11>+12 * u A B[3]) \\
& * e A B[3]+(-6+6 * u A B[4]) * e A B[4] .
\end{aligned}
$$

Since $u t[1]=u A B[1], u t[4]=u A B[2], u t[7]=u A B[3]$ and $u t[13]=u A B[4]$, we can compare the coefficients of these in (5) and (7) and obtain the following relations among the CECs corresponding to ternary and binary systems.

$$
\begin{aligned}
& e A B[1]=e t[1]-(3 / 2)^{*} \operatorname{et}[8]+(8 / 3) * \operatorname{et}[15], \\
& e A B[2]=\operatorname{et}[4]-2 * \operatorname{et}[9]+2 * \operatorname{et}[16], \\
& e A B[3]=e t[7]-(1 / 4) * \operatorname{et}[14], \\
& e A B[4]=\operatorname{et}[13] .
\end{aligned}
$$

We can obtain relations among the CECs corresponding to ternary and binary $A-C$ and $B-C$ subsystems in a similar fashion. The bases for $A-C$ and $B-C$ subsystems are found by setting respectively $p_{i}^{B}=0$ and $p_{i}^{A}=0$ in (2). For the subsystem $A-C$,

$$
\left[\begin{array}{c}
1 \\
s 1 i
\end{array}\right]=\left[\begin{array}{cc}
1 & 1 \\
-1 & 0
\end{array}\right]\left[\begin{array}{c}
p_{i}^{A} \\
p_{i}^{C}
\end{array}\right] .
$$

For the subsystem $B-C$,

$$
\left[\begin{array}{c}
1 \\
s 1 i
\end{array}\right]=\left[\begin{array}{ll}
1 & 1 \\
1 & 0
\end{array}\right]\left[\begin{array}{c}
p_{i}^{B} \\
p_{i}^{C}
\end{array}\right] .
$$

The relations among the CECs in both these cases are given below.

$$
\begin{aligned}
\operatorname{eAC}[1]= & \operatorname{et}[1]+(3 / 2) * \operatorname{et}[2]+(9 / 4) * \operatorname{et}[3]+3 * \operatorname{et}[8]+ \\
& (9 / 2) * \operatorname{et}[10]+9 * \operatorname{et}[11]+(27 / 2) * \operatorname{et}[12]+(3 / 2) \\
& * \operatorname{et}[15]+(9 / 2) * \operatorname{et}[17]+(27 / 2) * \operatorname{et}[18], \\
\operatorname{eAC}[2]= & \operatorname{et}[4]+(3 / 2) * \operatorname{et}[5]+(9 / 4) * \operatorname{et}[6]+4 * \operatorname{et}[9]+6 \\
& * \operatorname{et}[10]+9 * \operatorname{et}[12]+2 * \operatorname{et}[16]+3 * \operatorname{et}[17]+9 * \\
& \operatorname{et}[18], \\
\operatorname{eAC}[3]= & \operatorname{et}[7]+(3 / 2) * \operatorname{et}[8]+(3 / 2) * \operatorname{et}[9]+(9 / 4) * \operatorname{et}[10]+ \\
& (9 / 4) * \operatorname{et}[11]+(27 / 8) * \operatorname{et}[12]+(1 / 2) * \operatorname{et}[14]+ \\
& (3 / 2) * \operatorname{et}[15]+(3 / 2) * \operatorname{et}[16]+(27 / 8) * \operatorname{et}[17]+ \\
& (27 / 4) * \operatorname{et}[18], \\
\operatorname{eAC}[4]= & \operatorname{et}[13]+(3 / 2) * \operatorname{et}[14]+(9 / 4) * \operatorname{et}[15]+(9 / 4) * \\
& \operatorname{et}[16]+(27 / 8) * \operatorname{et}[17]+(81 / 16) * \operatorname{et}[18] .
\end{aligned}
$$

$$
\begin{aligned}
e B C[1]= & \operatorname{et}[1]-(3 / 2) * \operatorname{et}[2]+(9 / 4) * \operatorname{et}[3]+3 * \operatorname{et}[8]- \\
& (9 / 2) * \operatorname{et}[10]-9 * \operatorname{et}[11]+(27 / 2) * \operatorname{et}[12]+(3 / 2) * \\
& \operatorname{et}[15]-(9 / 2) * \operatorname{et}[17]+(27 / 2) * \operatorname{et}[18],
\end{aligned}
$$




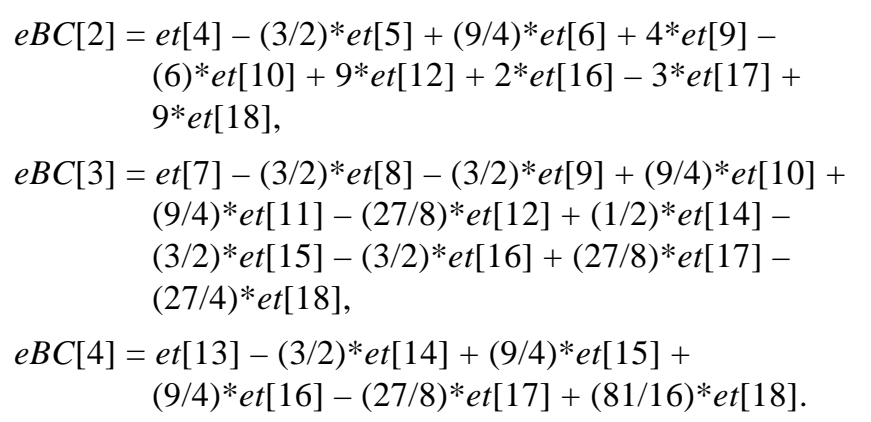

There are three first neighbour pair CECs for a ternary system (et[1] to et [3]) and three CECs arise from binary systems $(e A B[1], e A C[1]$ and $e B C[1])$. The same is true for second neighbour pair CECs. However, there are six triangle CECs for a ternary system (et[7] to et [12]) while only three CECs arise from binary systems ( $e A B[3]$, $e A C[3]$ and $e B C[3])$. The additional three CECs represent purely ternary triangle interactions. The same is true for tetrahedral CECs. There are three additional CECs representing purely ternary tetrahedral interactions. Hence, there are a total of six purely ternary interactions, the CECs for which must be selected to complement the component binary interactions in a mutually consistent fashion. To achieve this, we note that the system of equations composed of (8), (11) and (12), consists of twelve equations in eighteen unknowns. The six purely ternary variables can be found from the null space vectors of the coefficient matrix. We treat the triangle and tetrahedral interactions separately. Considering triangle interactions first, we obtain from (8), (11) and (12)

$$
\begin{aligned}
e A B[3]= & \operatorname{et}[7], \\
e A C[3]= & \operatorname{et}[7]+(3 / 2) * \operatorname{et}[8]+(3 / 2) * \operatorname{et}[9]+(9 / 4) * \\
& \operatorname{et}[10]+(9 / 4) * \operatorname{et}[11]+(27 / 8) * \operatorname{et}[12], \\
& \text { eBC[3]= } \operatorname{et}[7]-(3 / 2) * \operatorname{et}[8]-(3 / 2) * \operatorname{et}[9]+(9 / 4) * \\
& \operatorname{et}[10]+(9 / 4) * \operatorname{et}[11]-(27 / 8) * \operatorname{et}[12] .
\end{aligned}
$$

The null space for the above system of equations is given by the three vectors $\{0,-9 / 8,-9 / 8,0,0,1\},\{0,0$, $0,-1,1,0\}$ and $\{0,-1,1,0,0,0\}$. Thus, the combinations of et $[i]$ which should be used for the three purely ternary triangle interactions are as follows.

$$
\begin{aligned}
& e A B C[1]=-(9 / 8) * e t[8]-(9 / 8) * e t[9]+e t[12], \\
& e A B C[2]=-e t[10]+e t[11], \\
& e A B C[3]=-e t[8]+\operatorname{et}[9] .
\end{aligned}
$$

A similar analysis yields the following combinations of $e t[i]$ for the three purely ternary tetrahedral interactions.

$$
\begin{aligned}
& e A B C[4]=-(9 / 8) * e t[15]-(9 / 8) * \operatorname{et}[16]+\operatorname{et}[18], \\
& e A B C[5]=-(9 / 4) * \operatorname{et}[14]+\operatorname{et}[17], \\
& e A B C[6]=-\operatorname{et}[15]+e t[16] .
\end{aligned}
$$

Hence, the eighteen equations in (8), (11), (12), (14) and (15) form an augmented system of equations relating the CECs corresponding to the binary systems and their ternary counterparts. In order to obtain the CECs in the ternary basis from an a priori knowledge of CECs in the component binary subsystem bases (as happens during the development of databases such that they are consistent with subsystem data), the above augmented system of equations can be solved for et [i], the solutions of which are given below.

$$
\begin{aligned}
& e t[1]=e A B[1]+(113728 * e A B[4]) / 344763-(54 * \\
& e A B C[1]) / 113-(3 * e A B C[3]) / 4+(9120 * e A B C[4]) / \\
& 12769+(4 * e A B C[6]) / 3+(8 * e A C[3]) / 113- \\
& (56864 * e A C[4]) / 344763-(8 * e B C[3]) / 113- \\
& (56864 * e B C[4]) / 344763 \text {, } \\
& e t[2]=2 * e A B[3]-(3 * e A B C[2]) / 2+(6 * e A B C[5]) / 97+ \\
& e A C[1] / 3-e A C[3]+(53 * e A C[4]) / 97- \\
& e B C[1] / 3-e B C[3]-(53 * e B C[4]) / 97 \text {, } \\
& e t[3]=(-4 * e A B[1]) / 9-(2661280 * e A B[4]) / 3102867- \\
& (120 * e A B C[1]) / 113+e A B C[3]-(17848 * \\
& e A B C[4]) / 38307-(7 * e A B C[6]) / 27+(2 * e A C[1]) / 9- \\
& (248 * e A C[3]) / 339+(1330640 * e A C[4]) / 3102867+ \\
& (2 * e B C[1]) / 9+(248 * e B C[3]) / 339+ \\
& (1330640 * e B C[4]) / 3102867 \text {, } \\
& e t[4]=e A B[2]+(39296 * e A B[4]) / 114921- \\
& (72 * e A B C[1]) / 113+e A B C[3]+(5832 * e A B C[4]) / \\
& 12769-e A B C[6]+(32 * e A C[3]) / 339- \\
& (19648 * e A C[4]) / 114921-(32 * e B C[3]) / 339- \\
& \text { (19648*eBC[4])114921, } \\
& e t[5]=\left(8^{*} e A B[3]\right) / 9+2 * e A B C[2]-\left(8^{*} e A B C[5]\right) / 97+ \\
& e A C[2] / 3-(4 * e A C[3]) / 9+(140 * e A C[4]) / 873- \\
& e B C[2] / 3-(4 * e B C[3]) / 9-(140 * e B C[4]) / 873 \text {, } \\
& e t[6]=(-4 * e A B[2]) / 9-(239296 * e A B[4]) / 344763- \\
& (32 * e A B C[1]) / 113-(4 * e A B C[3]) / 3-(1024 * \\
& e A B C[4]) / 12769+(2 * e A C[2]) / 9-(560 * \\
& e A C[3]) / 1017+(119648 * e A C[4]) / 344763+(2 * \\
& e B C[2]) / 9+(560 * e B C[3]) / 1017+(119648 * e B C[4]) / \\
& 344763 \text {, } \\
& e t[7]=e A B[3]-(9 * e A B C[5]) / 97+(4 * e A C[4]) / 291- \\
& (4 * e B C[4]) / 291 \text {, } \\
& e t[8]=\left(12416^{*} e A B[4]\right) / 114921-\left(36^{*} e A B C[1]\right) / 113- \\
& e A B C[3] / 2-(1152 * e A B C[4]) / 12769+\left(16^{*}\right. \\
& e A C[3]) / 339-(6208 * e A C[4]) / 114921-\left(16^{*}\right. \\
& e B C[3]) / 339-\left(6208^{*} e B C[4]\right) / 114921 \text {, } \\
& e t[9]=\left(12416^{*} e A B[4]\right) / 114921-\left(36^{*} e A B C[1]\right) / 113+ \\
& e A B C[3] / 2-(1152 * e A B C[4]) / 12769+\left(16^{*}\right. \\
& e A C[3]) / 339-(6208 * e A C[4]) / 114921-\left(16^{*}\right. \\
& e B C[3]) / 339-(6208 * e B C[4]) / 114921 \text {, } \\
& e t[10]=(-2 * e A B[3]) / 9-e A B C[2] / 2-(6 * e A B C[5]) / 97+ \\
& e A C[3] / 9-(89 * e A C[4]) / 873+e B C[3] / 9+ \\
& (89 * e B C[4]) / 873 \text {, } \\
& e t[11]=(-2 * e A B[3]) / 9+e A B C[2] / 2-(6 * e A B C[5]) / 97+ \\
& e A C[3] / 9-(89 * e A C[4]) / 873+e B C[3] / 9+ \\
& (89 * e B C[4]) / 873 \text {, }
\end{aligned}
$$




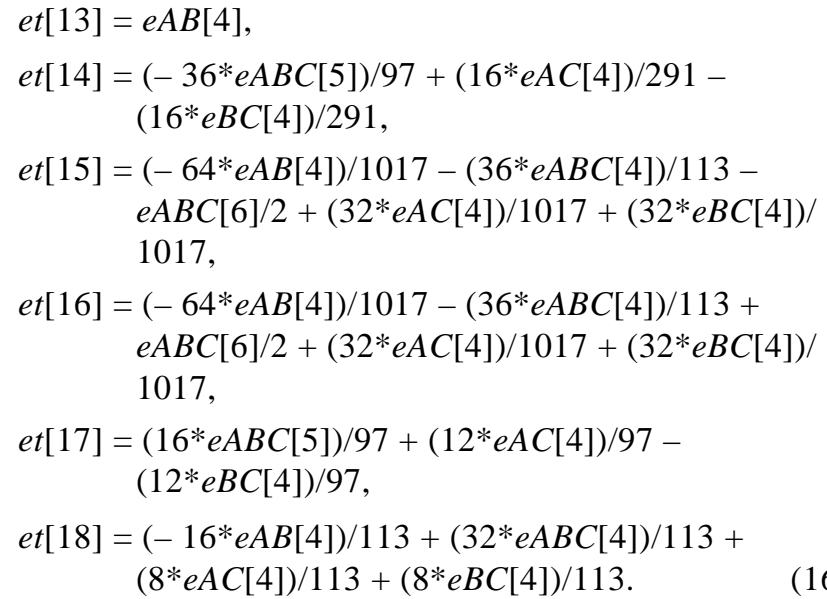

In case the purely ternary triangle and tetrahedral CECs defined in (14) and (15) vanish, we obtain from (16), a set of ternary CECs which are functions only of the binary subsystem CECs. Thus, we have given a procedure for obtaining the complete set of ternary basis CECs in terms of their component subsystem CECs and an independent set of pure ternary CECs. It may be added that such procedures can be generalized to higher order systems in a straightforward manner.

\section{Basis transformations}

It must, however, be noted that the CECs for subsystems are defined in different bases as given in (6), (9) and (10). It is desirable to have CECs of all subsystems in a standard orthogonal basis. Thus, we must be able to evaluate CECs in one basis from those in another basis. In the particular example considered above, we require transformations from binary orthogonal basis [e.g. in (6)] to nonorthogonal ones, such as those for $A-C$ [in (9)] and $B-C$ [in (10)] systems. In addition, we require transformations from orthogonal basis to dilute solution bases, as explained below. An orthogonal basis must be generally selected for determining the CECs for any materials system. However, such a basis is not ideal for finding internal equilibria of very dilute solutions. This can be taken care of by selecting different non-orthogonal bases for each solvent, e.g. for $C$-solvent the basis given in (10) may be chosen in the $B-C$ subsystem. This results in the probabilities of all clusters constituted entirely of solute (or B) atoms being directly equal to a corresponding correlation function in this basis. It may be pointed out that such a choice of dilute solution bases is useful in obtaining certain analytical results for the values of correlation functions and other thermodynamic functions of interest in the infinite dilution limit. These results will be presented in another communication. Given below are the details of transformations of CECs (or correlation functions) from one basis to another.

Since any function of configuration (such as internal energy) expressed as in (3) should remain invariant under these transformations, we can write

$$
U=\sum_{i=1}^{M} e_{i}^{\mathrm{I}} m_{i} u_{i}^{\mathrm{I}}=\sum_{i=1}^{M} e_{i}^{\mathrm{II}} m_{i} u_{i}^{\mathrm{II}}
$$

in which the superscripts I and II refer to two bases, respectively and $M$ the number of independent correlation functions including point and empty correlation functions. This can be represented in matrix notation as

$$
\left\{e^{\mathrm{I}}\right\}_{1 \times M^{*}}\left[m_{\mathrm{D}}\right]_{M \times M^{*}}\left[u^{\mathrm{I}}\right]_{M \times 1}=\left\{e^{\mathrm{II}}\right\}_{1 \times M^{*}}\left[m_{\mathrm{D}}\right]_{M \times M^{*}}\left[u^{\mathrm{II}}\right]_{M \times 1} .
$$

It should be noted that $\left[m_{\mathrm{D}}\right]_{M \times M}$ is a diagonal matrix, whose elements are the multiplicities of the (sub)motifs and braces represent a row vector. Further, the cluster probabilities $\rho$ also should remain invariant under these transformations. Thus, in matrix notation,

$$
[\rho]_{M \times 1}=\left[B^{\mathrm{I}}\right]_{M \times M} \cdot\left[u^{\mathrm{I}}\right]_{M \times 1}=\left[B^{\mathrm{II}}\right]_{M \times M^{*}} \cdot\left[u^{\mathrm{II}}\right]_{M \times 1},
$$

where $[B]$ are the square transformation matrices corresponding to any of the $M$ linearly independent relations among cluster probabilities and correlation functions and can be found by using the $R$ matrix defined earlier.

In the case of a $K$ component system, the $[B]$ matrices can be obtained by selecting any $(K-1)$ equations from the set of equations for cluster variables corresponding to each of the symmetry-wise distinct submotifs, along with the one corresponding to the empty cluster, $\rho^{0}=1$. From (19), it follows that

$$
\left[u^{\mathrm{II}}\right]=\left[B^{\mathrm{II}}\right]^{-1} \cdot\left[B^{\mathrm{I}}\right] \cdot\left[u^{\mathrm{I}}\right] .
$$

It may be noted that the matrix product $\left[B^{\mathrm{II}}\right]^{-1} \cdot\left[B^{\mathrm{I}}\right]$ remains unchanged irrespective of the choice of this set of equations, even though the individual $[B]$ matrices in both the bases may differ. Substituting for $\left[u^{\mathrm{II}}\right]$ from (20) in (18) leads to

$$
\left\{e^{\mathrm{I}}\right\}=\left\{e^{\mathrm{II}}\right\} \cdot\left[m_{\mathrm{D}}\right] \cdot\left[B^{\mathrm{II}}\right]^{-1} \cdot\left[B^{\mathrm{I}}\right] \cdot\left[m_{\mathrm{D}}\right]^{-1},
$$

or, equivalently

$$
\left[e^{\mathrm{I}}\right]=\left[m_{\mathrm{D}}\right]^{-1} \cdot\left[B^{\mathrm{I}}\right]^{T} \cdot\left[B^{\mathrm{II}}\right]^{-T} \cdot\left[m_{\mathrm{D}}\right] \cdot\left[e^{\mathrm{II}}\right],
$$

in which the superscripts $-1, T$ and $-T$ represent respectively, the inverse, transpose and transpose of inverse of the matrices concerned.

We shall illustrate this procedure for transforming binary CECs in orthogonal basis [given in (6), to be represented by superscript II] to a nonorthogonal basis [given in (10), to be represented by superscript I]. To find the $B$ matrices, we note that $R^{\mathrm{I}}$ and $R^{\mathrm{II}}$ are given by

$$
R^{\mathrm{I}}=\left[\begin{array}{cc}
0 & 1 \\
1 & -1
\end{array}\right] \text {, }
$$

and

$$
R^{\mathrm{II}}=\left[\begin{array}{cc}
1 / 2 & -1 / 2 \\
1 / 2 & 1 / 2
\end{array}\right] .
$$


We now express the cluster variables corresponding to first neighbour $A A$ pairs, second neighbour $A A$ pairs, triangle clusters of $A A A$ triplet, tetrahedral cluster of $A A A A$ quadruplet, fraction of $A$ atoms and the empty cluster, respectively in terms of correlation functions in each basis. This yields the following $B$ matrices.

$$
\begin{aligned}
B^{\mathrm{I}} & =\left[\begin{array}{cccccc}
1 & 0 & 0 & 0 & 0 & 0 \\
0 & 1 & 0 & 0 & 0 & 0 \\
0 & 0 & 1 & 0 & 0 & 0 \\
0 & 0 & 0 & 1 & 0 & 0 \\
0 & 0 & 0 & 0 & 1 & 0 \\
0 & 0 & 0 & 0 & 0 & 1
\end{array}\right], \\
B^{\mathrm{II}} & =\left[\begin{array}{ccccccc}
1 / 4 & 0 & 0 & 0 & -1 / 2 & 1 / 4 \\
0 & 1 / 4 & 0 & 0 & -1 / 2 & 1 / 4 \\
1 / 4 & 1 / 8 & -1 / 8 & 0 & -3 / 8 & 1 / 8 \\
1 / 4 & 1 / 8 & -1 / 4 & 1 / 16 & -1 / 4 & 1 / 16 \\
0 & 0 & 0 & 0 & -1 / 2 & 1 / 2 \\
0 & 0 & 0 & 0 & 0 & 1
\end{array}\right] .
\end{aligned}
$$

The diagonal matrix of multiplicities (given above) is written as

$$
m_{\mathrm{D}}=\left[\begin{array}{cccccc}
4 & 0 & 0 & 0 & 0 & 0 \\
0 & 3 & 0 & 0 & 0 & 0 \\
0 & 0 & 12 & 0 & 0 & 0 \\
0 & 0 & 0 & 6 & 0 & 0 \\
0 & 0 & 0 & 0 & 1 & 0 \\
0 & 0 & 0 & 0 & 0 & 1
\end{array}\right] .
$$

Thus, from (22), we obtain the following relations among CECs.

$$
\begin{aligned}
e^{\mathrm{I}}[1]= & \left(16 * e^{\mathrm{II}}[1]-96 * e^{\mathrm{II}}[3]+2 *\left(96 * e^{\mathrm{II}}[3]-\right.\right. \\
& \left.\left.192 * e^{\mathrm{II}}[4]\right)+480 * e^{\mathrm{II}}[4]\right) / 4, \\
e^{\mathrm{I}}[2]= & \left(12 * e^{\mathrm{II}}[2]+48 * e^{\mathrm{II}}[3]+48 * e^{\mathrm{II}}[4]\right) / 3, \\
e^{\mathrm{I}}[3]= & \left(-96 * e^{\mathrm{II}}[3]-192 * e^{\mathrm{II}}[4]\right) / 12,
\end{aligned}
$$

$$
\begin{aligned}
e^{\mathrm{I}}[4]= & 16 * e^{\mathrm{II}}[4], \\
e^{\mathrm{I}}[5]= & 16 * e^{\mathrm{II}}[1]+12 * e^{\mathrm{II}}[2]-72 * e^{\mathrm{II}}[3]-3 *\left(96 * e^{\mathrm{II}}[3]-\right. \\
& \left.192 * e^{\mathrm{II}}[4]\right)-336^{*} e^{\mathrm{II}}[4]-2 *\left(12 * e^{\mathrm{II}}[2]-\right. \\
& \left.48 * e^{\mathrm{II}}[3]+48 * e^{\mathrm{II}}[4]\right)-2 *\left(16 * e^{\mathrm{II}}[1]-\right. \\
& \left.96 * 6^{\mathrm{II}}[3]+96 * e^{\mathrm{II}}[4]\right)-2 * e^{\mathrm{II}}[5], \\
e^{\mathrm{I}}[6]= & 4 * e^{\mathrm{II}}[1]+3 * e^{\mathrm{II}}[2]+12 * e^{\mathrm{II}}[3]+6 * e^{\mathrm{II}}[4]+ \\
& e^{\mathrm{II}}[5]+e^{\mathrm{II}}[6] .
\end{aligned}
$$

Similar procedures can be adopted for transformation of correlation functions from any basis to another.

\section{Conclusions}

We have given a procedure for expressing the CECs in a multicomponent system basis in terms of those in the constituent subsystems bases and the independent CECs representing the specific multicomponent interactions. This procedure is illustrated with reference to disordered ternary $b c c$ structure. Further, we have given the details of transformations required for obtaining CECs from one basis to another in a given system.

\section{References}

Asta M, de Fontaine D and McCormack R C 1993 Phys. Rev. B48 748

van Baal C M 1973 Physica 64571

de Fontaine D 1979 Solid State Phys. 3473

de Fontaine D 1994 Solid State Phys. 4733

Inden G and Pitsch W 1991 Materials science and technology (ed) P Haasen (Weinheim: VCH Verlagsgesellschaft) Vol. 5, p. 497

Kikuchi R 1951 Phys. Rev. 81988

Kikuchi R 1977 Acta Metall. 25195

Kikuchi R, de Fontaine D, Murakami M and Nakamura T 1977 Acta Metall. 25207

Sanchez J M and de Fontaine D 1978 Phys. Rev. B17 2926

Sanchez J M, Ducastelle F and Gratias D 1984 Physica A128 334

Saunders N and Miodownik A P 1998 CALPHAD (Calculation of phase diagrams): A comprehensive guide (Oxford: Pergamon)

Wolverton C and de Fontaine D 1994 Phys. Rev. B49 8627 